\title{
REVIEWERS
}

\section{Guest Reviewers, Journal of Classification Volume 28(1) 2011, Special Issue}

In addition to the members of the Editorial Board, the individuals listed below refereed manuscripts that were submitted to the journal for Volume 28(1) 2011, Special Issue. Their assistance is gratefully acknowledged.

\author{
Amjad Al-Nasser \\ Mohammed Al-Rawwash \\ Tomas Aluja-Banet \\ Pietro Amenta \\ Eric Beh \\ Eugenio Brentari \\ Enrico Ciavolino \\ Luigi D'Ambra \\ Jules de Tibeiro \\ Michele Gallo \\ Boris Goldengorin \\ Ron Kenett \\ Anita van der Kooij \\ Rosaria Lombardo \\ Smail Mahdi \\ Jacqueline Meulman \\ John Mingers \\ El Mostafa Qannari \\ Robert Sabatier \\ Massimo Squillante \\ Yoshio Takane
}

